\title{
Antidepressant action through gene regulation
}

\author{
A recent paper in the Journal of \\ Neuroscience has shown that inhibi- \\ tors of histone deacetylases (HDACs) \\ - enzymes that affect the acetylation \\ status of histones and regulate the \\ remodelling of chromatin - have \\ antidepressant actions. \\ Although currently used anti-
} depressants rapidly modulate monoaminergic systems in the brain, the emergence of their moodelevating effects requires several weeks of administration, which suggests that altered gene expression is involved in antidepressant action. So, compounds that modulate the epigenetic regulation of gene expression, such as HDAC inhibitors, might have antidepressant actions.

Nestler and colleagues used a mouse model of chronic social defeat to investigate histone acetylation in depression and the effect of HDAC inhibitors in the nucleus accumbens, which is a brain region implicated in the development of depression and antidepressant action.

Immunohistochemical analysis showed that, although acetylation levels of histone $\mathrm{H} 3$ at lysine residue 14 (acH3K14) decreased transiently by $\sim 50 \% 1$ hour after the final stress event, there was an increase at 24 hours and 10 days. Expression levels of HDAC2, but not of HDAC1 or HDAC3, were decreased 24 hours and 10 days after the final stress event, suggesting that this could mediate the increase in acH3K14 levels. A similar increase in acH3K14 levels, accompanied by a decrease in HDAC2 levels, was present in post-mortem samples of the nucleus accumbens from depressed humans.

Infusion of the HDAC inhibitors vorinostat (a class I and II HDAC inhibitor) or MS-275 (a class I HDAC inhibitor) into the nucleus accumbens of mice that were subjected to chronic social-defeat stress reversed stress-induced social avoidance and increased the amount of time that the mice spent socially interacting. In forced-swim tests, which are often used as an acute screen for antidepressants, both inhibitors showed antidepressant-like effects but had no effect on anxietylike behaviour.

As chronic social-defeat stress leads to distinctive patterns of gene expression in the nucleus acumbens, which can almost be normalized by fluoxetine treatment, the authors tested the effects of MS-275 on gene expression using microarray analysis. Like fluoxetine, MS-275 mostly reversed stress-induced genomic changes, and both fluoxetine and MS-275 treatment caused similar changes in the expression patterns of many genes. The regulation of certain genes by chronic stress was reversed by MS-275 but not fluoxetine, which might reveal new

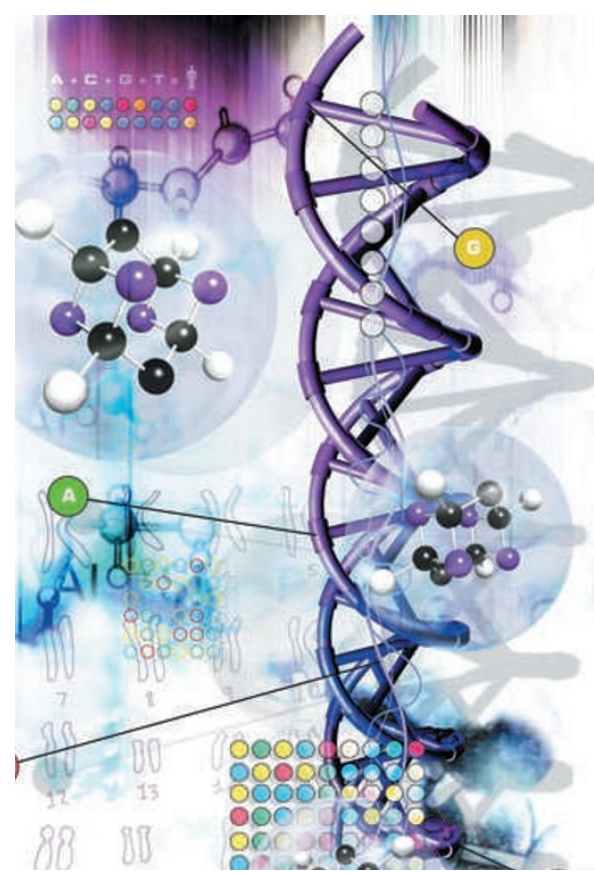

targets for antidepressant action. These included genes encoding gap junction membrane channel protein $\alpha 5$ (which is involved in gap junction formation), discs large-associated protein 1 (which assembles postsynaptic density complexes) and the $\alpha_{1 \alpha}$-adrenergic receptor.

So, although selectivity and delivery issues remain to be resolved, HDAC inhibitors, which are in clinical trials for cancer indications, might also have therapeutic potential in depression.

Charlotte Harrison

\section{ORIGINAL RESEARCH PAPER}

Covington, H. E.et al. Antidepressant actions of histone deacetylase inhibitors. J. Neurosci. 29, 11451-11460 (2009)

FURTHER READING Kazantsev, A. G. \& Thompson, L. M. Therapeutic application of histone deacetylase inhibitors for central nervous system disorders. Nature Rev. Drug Discov. 7, 854-868 (2008) 